\title{
Hydrodynamic nucleation of quantized vortex pairs in a polariton quantum fluid
}

\author{
Gaël Nardin ${ }^{\star}$, Gabriele Grosso, Yoan Léger, Barbara Piẹtka†', François Morier-Genoud \\ and Benoît Deveaud-Plédran
}

Quantized vortices appear in quantum gases at the breakdown of superfluidity. In liquid helium and cold atomic gases, they have been indentified as the quantum counterpart of turbulence in classical fluids. In the solid state, composite light-matter bosons known as exciton polaritons have enabled studies of non-equilibrium quantum gases and superfluidity. However, there has been no experimental evidence of hydrodynamic nucleation of polariton vortices so far. Here we report the experimental study of a polariton fluid flowing past an obstacle and the observation of nucleation of quantized vortex pairs in the wake of the obstacle. We image the nucleation mechanism and track the motion of the vortices along the flow. The nucleation conditions are established in terms of local fluid density and velocity measured on the obstacle perimeter. The experimental results are successfully reproduced by numerical simulations based on the resolution of the Gross-Pitaevskii equation.

H ydrodynamic instabilities in classical fluids were studied in the pioneering experiments of Bénard in the 1910's. Convective Bénard-Rayleigh flows and Bénard-Von Kármán streets are now well known examples in nonlinear and chaos sciences $^{1}$. In conventional fluids, the flow around an obstacle is characterized by the dimensionless Reynolds number $\operatorname{Re}=v R / v$, with $v$ and $v$ the fluid velocity and dynamical viscosity, respectively, and $R$ the diameter of the obstacle. When increasing the Reynolds number, laminar flow, stationary vortices, Bénard-Von Kármán streets of moving vortices and fully turbulent regimes are successively observed in the wake of the obstacle ${ }^{1}$.

In quantum fluids, such as liquid helium or atomic BoseEinstein condensates, quantum turbulence has long been predicted to appear at the breakdown of superfluidity ${ }^{2-8}$. In superfluid systems, the Reynolds number cannot be defined owing to the absence of viscosity. However, quantum turbulence, in the form of quantized vortices, appears simultaneously with dissipation and drag on the obstacle once a critical velocity is exceeded. This critical velocity is predicted to be lower than the Landau criterion for superfluidity far from the obstacle, because of a local increase of the fluid velocity in the vicinity of the impenetrable obstacle $e^{2,4,5}$.

Experimental evidence has been given for the appearance of a drag force or heat above some critical velocity in superfluid helium $^{5}$ and atomic Bose-Einstein condensates ${ }^{9,10}$. In stirred atomic gases, vortex lattices appear above a critical stirring frequency ${ }^{11-13}$, analogously to the rotating bucket experiments originally performed with superfluid helium ${ }^{14}$. Irregular vortex tangle patterns were also observed under an external oscillating perturbation, indicating the presence of turbulence in the atomic cloud $^{15}$. Finally, vortex nucleation has been reported in the wake of a blue-detuned laser moving above a critical velocity through the condensate ${ }^{16,17}$. However, no experiment has yet allowed the imaging of the hydrodynamic nucleation mechanism with phase resolution.

The recent demonstration of superfluidity in an exciton polariton gas in a semiconductor microcavity ${ }^{18}$ offers a very advantageous tool to explore quantum turbulence. Exciton polaritons are composite bosons resulting from the strong coupling between the excitonic resonance of a semiconductor quantum well and the microcavity electromagnetic field ${ }^{19}$. Their dual light-matter nature gives them many advantages. Thanks to a one-to-one coupling to the extra-cavity field, with conservation of in-plane momentum and energy, exciton polaritons can be easily optically injected, manipulated ${ }^{20,21}$, and detected. Thanks to their excitonic part, polaritons can interact with each other, leading to spectacular nonlinear behaviours ${ }^{22}$. Since the demonstration of polariton Bose-Einstein condensation ${ }^{23}$, numerous experimental and theoretical works have explored the rich physics underlying the driven-dissipative character of these non-equilibrium condensates ${ }^{24-27}$.

In particular, observation of quantized vortices has been reported in polariton degenerate gases in several configurations. In a condensed phase, they are the result of the interplay between the non-equilibrium nature of the condensate and natural disorder present in the cavity ${ }^{28-30}$. Quantized vortices have also been optically imprinted in optical parametric oscillator (OPO) configurations ${ }^{31,32}$. On the other hand, frictionless flow of a polariton wave packet has been reported in a triggered OPO scheme $\mathrm{e}^{33}$, and the superfluid regime has been demonstrated in a resonantly injected polariton fluid ${ }^{18,34}$. In these last papers, depending on the fluid velocity, superfluid or Čerenkov regimes were observed as the steady polariton flow scatters on a defect. However, the experimental observation of the hydrodynamic nucleation of polariton vortices has not been reported so far.

In this Article, we make use of a polariton fluid to demonstrate the nucleation of quantized vortex pairs in the wake of an obstacle. High-resolution experimental snapshots of the polariton fluid density and phase allow the imaging of the nucleation mechanism and the migration of the vortices in the microcavity plane. The nucleation conditions are analysed in terms of the local fluid velocity and sound velocity on the obstacle perimeter, and qualitatively reproduced using numerical simulations based on the resolution of the generalized Gross-Pitaevskii equation.

Laboratoire d'Optoélectronique Quantique, École Polytechnique Fédérale de Lausanne (EPFL), Station 3, CH-1015 Lausanne, Switzerland. †Present address: Faculty of Physics, Institute of Experimental Physics, University of Warsaw, ul. Hoża 69, 00-681 Warsaw, Poland. *e-mail: gael.nardin@epfl.ch. 


\section{Superfluidity and turbulence in microcavities}

Similarly to conventional superfluids, quantum turbulence is expected to appear in microcavities at the breakdown of superfluidity ${ }^{35,36}$. As the sound velocity in the system is decreased, hydrodynamic nucleation of quantized vortices occurs when the local fluid velocity in the vicinity of the obstacle becomes larger than the sound velocity. A Čerenkov regime, accompanied by soliton lines, follows when the velocity of the fluid far from the obstacle becomes greater than the speed of sound ${ }^{36}$. A crucial point is that the excitation laser imprints its phase on the polariton fluid, preventing the formation of non-trivial phase structures such as vortices. To overcome this issue, an abrupt switch-off of the excitation laser ${ }^{35}$ or a non-uniform spatial pump profile ${ }^{36}$ have been proposed.

In our experiment, we use a 3 ps-long pulse to create a polariton wave packet, which can then evolve freely in the microcavity plane during the polariton lifetime (measured characteristic decay time: $\tau \sim 15 \mathrm{ps}$ ), providing a sufficiently long time window during which quantum turbulence can form. We resonantly inject polaritons in the lower polariton branch with an initial in-plane momentum imposed by the pump of $k_{\|}=1.2 \mu \mathrm{m}^{-1}$ (in Fig. $1 \mathrm{~b}$ the yellow circle indicates the pump extension in energy and momentum). We allow the wave packet to scatter on a structural defect of $\sim 5 \mu \mathrm{m}$ transverse size (Fig. 1a). We have developed a time- and phaseresolved imaging set-up, based on a Mach-Zehnder interferometer (see Methods), to observe the dynamics of the scattering on a picosecond timescale and track the formation and migration of vortices in the turbulent flow. Quantized vortices are characterized by a density minimum at the core and a $2 \pi$-phase rotation around the singularity, which results in a fork-like dislocation when interference takes place with a plane reference wave $e^{16,28}$.

As a result of polariton-polariton interactions, resonantly pumping the polariton branch strongly modifies the excitation spectrum $^{34}$. These interactions result in a blue-shift of the dispersion curve (interaction energy) and a linearization of the dispersion (Bogoliubov spectrum) in the vicinity of the polariton gas. This allows one to define a sound velocity $c_{\mathrm{s}}$ for the polariton fluid that is dependent on the polariton density $n$, as $c_{\mathrm{s}}=\sqrt{n g / m_{\mathrm{LP}}}$ (where $g$ is the polariton-polariton interaction constant and $m_{\mathrm{LP}}$ the lower polariton effective mass), and can therefore be controlled by tuning the excitation power. Figure 1c shows the different shapes of the lower polariton dispersion (in a parabolic approximation) for a polariton population at $k_{\|}=1.2 \mu \mathrm{m}^{-1}$ (corresponding to a flow speed of $v=\hbar k_{\|} / m_{\mathrm{LP}}=1.13 \mu \mathrm{m} \mathrm{ps}^{-1}$ ). The dispersion curve changes from a fully parabolic dispersion in the low-density regime (black curve) to a superfluid dispersion (blue curve). On these curves, the injected polariton population is indicated by a black dot. In the low-density regime (black curve), the possibilities of elastic scattering due to disorder (the intersection with the dashed line) form the so-called Rayleigh ring in the two-dimensional momentum space. Under high excitation density (blue curve), superfluidity arises because the dispersion no longer offers the possibility of Rayleigh scattering. This prevents any interaction of the polariton fluid with disorder. This collapse of the Rayleigh ring has been experimentally demonstrated by Amo et al. ${ }^{33}$. In this case, the Landau criterion is fulfilled, as the fluid velocity is lower than the sound velocity. It corresponds to a Mach number (defined as the ratio of the fluid velocity $v$ over the sound speed $\left.c_{\mathrm{s}}\right)$ smaller than one $\left(v / c_{\mathrm{s}}<1\right)$. The red dispersion curve depicts a Čerenkov regime, where the fluid velocity is greater than the sound speed $\left(v / c_{\mathrm{s}}>1\right)$. The green dispersion curve shows an intermediate regime, corresponding to a Mach number of $v / c_{s}=1$. In such a case, the dispersion curve is flat on a finite distribution of wave vectors, offering a contiguous reciprocal space region in which Rayleigh scattering can occur. Using a pulsed excitation, we expect to pass through all these regimes (as schematically indicated by the black time arrow in Fig. 1c) after the polariton injection, leading to an extremely rich dynamics.

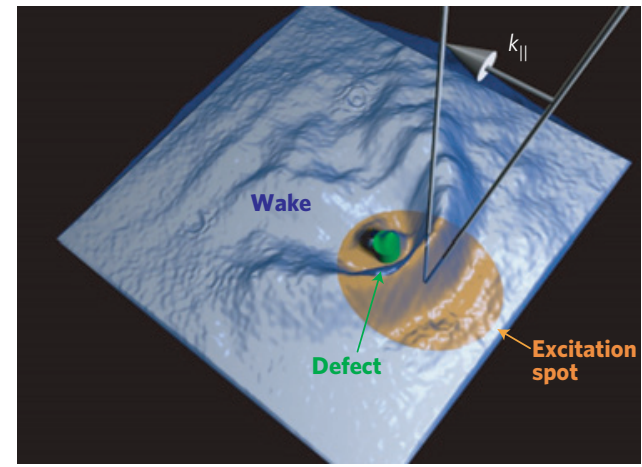

b

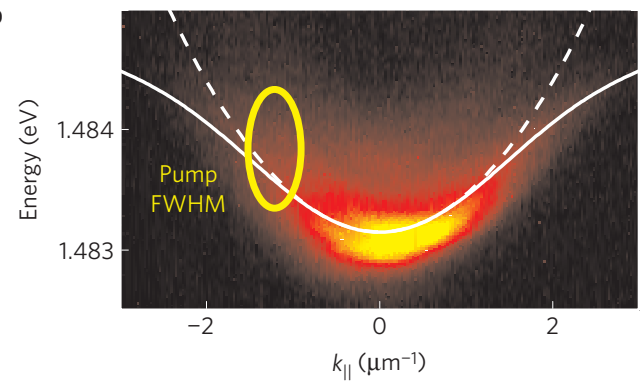

c

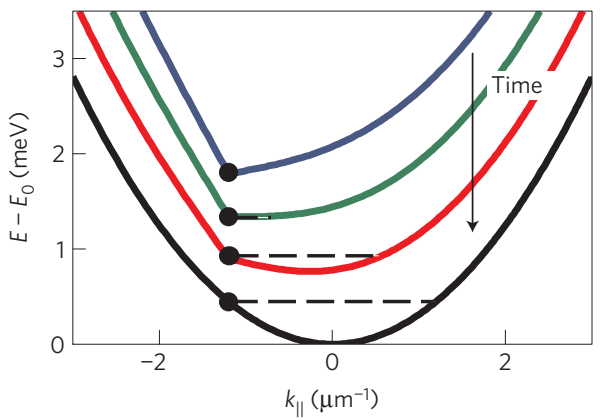

Figure 1 | Experimental scheme. a, Scheme of the experiment. A polariton wave packet is resonantly injected with an in-plane wave vector $k_{\|}$in front of a structural defect on which it scatters. b, Experimental polaritonic dispersion curve, under non-resonant pumping. The solid line indicates the theoretical lower polariton (LP) dispersion, and the dashed line the standard small momentum parabolic approximation of the LP branch. During the experiment, polaritons are resonantly injected in the lower polariton branch: the yellow circle indicates the energy and momentum extension of the pulsed pump. c, Theoretical dispersion curves. Under low excitation density, the system is in the linear regime and the dispersion is parabolic in the small momentum approximation (black curve). The polariton population is given by the black dot, and the intersection of the dashed line with the dispersion gives the possibility of scattering, which conserves energy. When increasing the pump power, the excitation spectrum is modified, going from a Čerenkov regime (red curve) to a superfluid regime (blue curve). The green curve shows an intermediate regime, for which the dispersion curve is flat on a finite distribution of wave vectors. The black arrow schematically shows the time evolution of the dispersion curve during the decay of the polariton population.

Interferometric measurements taken for different delays between the two Mach-Zehnder interferometer arms allow us to retrieve the dynamics of the polariton fluid (each measurement is therefore an integration over multiple experiments at a fixed delay - see Methods). In Fig. 2a we display the normalized polariton density integrated in the vicinity of the defect (red curve), and compare it to the normalized autocorrelation measurement of the laser (black curve). It shows that a significant fraction of the polariton population is still passing in the vicinity of the obstacle even when the excitation pulse has gone. 

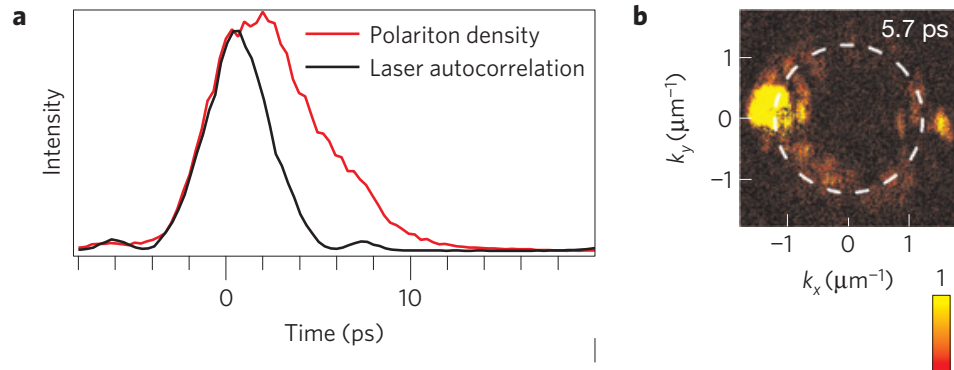
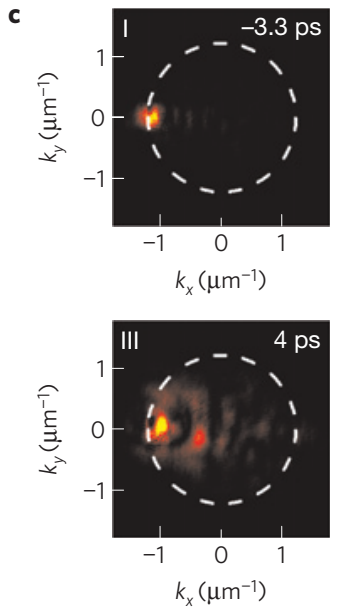
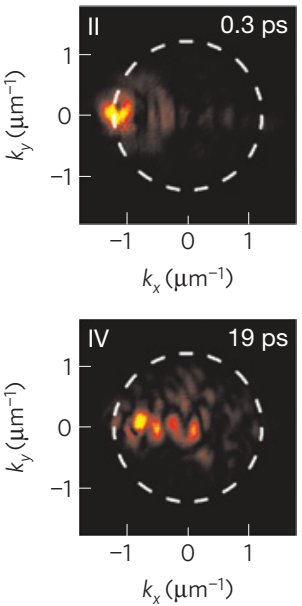

Figure 2 | Population dynamics. a, Red curve: measured polariton density, integrated in a small region in the vicinity of the obstacle, with respect to the interferometer delay. Black curve: autocorrelation of the pulsed excitation. The comparison of the red and black curves shows the time window on which the created polariton wave packet is allowed to evolve. b. Momentum space image of the emission under low excitation power. In this low excitation density regime, a Rayleigh ring is visible, as a result of the scattering of the polariton wave packet on the defect. The intensity of the Rayleigh ring is maximal 5.7 ps after the excitation pulse. Dashed line indicates the expected position of the Rayleigh ring in the low-density regime. c, Momentum space dynamics of the emission under high excitation power. The time-dependent modification of the excitation spectrum leads to a spreading of the polariton population inside the Rayleigh ring, and a general slowing down of the polariton propagation.

\section{Momentum space dynamics}

We first discuss the dynamics in momentum space. At low excitation pump power, the system is in the linear regime (black curve in Fig. 1c). In this case, a Rayleigh ring (Fig. 2b) appears as the result of elastic scattering of polaritons on surrounding disorder, and disappears with the polariton decay time. At high pump power, the time-dependent polariton density will make the dispersion curve vary with time, passing through the different cases depicted in Fig. 1c. The four panels of Fig. 2c show different snapshots of this evolution. At first the excitation laser creates a wave packet with an in-plane momentum of $k_{\|}=1.2 \mu \mathrm{m}^{-1}$ (Fig. $2 \mathrm{cI}$ ). Then, as the local polariton density decays, we observe the filling of the interior of the Rayleigh ring (Fig. 2cII-IV), resulting in an average slowing down of the wave packet. This behaviour can be qualitatively reproduced using the generalized Gross-Pitaevskii model (see Methods and Supplementary Information) and understood as follows: the decay of the polariton density is accompanied by a decrease of the sound velocity. When $v \sim c_{\mathrm{s}}$ (green dispersion curve in Fig. 1c), elastic scattering of the polariton wave packet on the environment fills a contiguous region inside the Rayleigh ring, relaxing the wave vector conservation rule. This is of crucial importance for the vortex nucleation mechanism, as vortices can appear only when a significant quantity of fluid has been slowed down behind the obstacle and a contiguous and sufficiently broad region of the reciprocal space provides the necessary wave vectors to form the rotating flow around the phase singularity.

\section{Vortex dynamics}

We now discuss the dynamics of the vortex nucleation and migration. Figure 3 displays experimental images of (1) the normalized polariton density, (2) the fringes of the interferogram (in a saturated colour scale, to track the fork-like dislocations), and (3) the phase of the polariton gas, for different times after the excitation pulse. The obstacle position is indicated by a green circle and the polariton flow is to the left. In the first column $(-0.7 \mathrm{ps})$, the phase structure is imposed by the excitation laser. The phase gradient allows one to extract the flow velocity, which is fairly homogeneous on the wave packet, and measured to be $1.1 \pm 0.2 \mu \mathrm{mps}^{-1}$, in agreement with the injected velocity of $1.13 \mu \mathrm{m} \mathrm{ps}^{-1}$. In the following, the fluid velocity will be specifically measured on two points of interest: behind the obstacle and on the equator of the obstacle perimeter. The corresponding measurement areas are indicated in Fig. 3a by a dashed white square and a plain white square, respectively. In the second column (1.3 ps), a low-density region appears in the wake of the defect, along with a curvature of the wavefront. The measurement of the phase gradient shows that the polariton flow slows down in the wake of the obstacle. The flow velocity behind the obstacle is shown in Fig. 4a (cyan curve). In the third column of Fig. 3 (3.7 ps), the flow velocity is measured to be $0.9 \pm 0.2 \mu \mathrm{m} \mathrm{ps}^{-1}$, dropping to $0.3 \pm 0.2 \mu \mathrm{mps}^{-1}$ in the fifth column $(9.3 \mathrm{ps})$, whereas the flow velocity measured on the obstacle perimeter, shown in Fig. 4a (black curve), remains above $0.95 \pm 0.35 \mu \mathrm{m} \mathrm{ps}^{-1}$ on this time range. As expected in a quantum fluid, where the circulation is quantized, the phase accumulation between the almost stationary wave behind the obstacle and the main flow is accompanied by the nucleation of quantized vortices ${ }^{3}$.

The nucleation of vortices can be observed in the third column (3.7 ps) of Fig. 3, where a tearing of the phase front is visible. Vortices are unambiguously identified by a minimum of density and a fork-like dislocation in the interferogram, accompanied by a phase singularity in the phase structure. They are indicated by white markers ( $x$ for vortex and + for anti-vortex) on the density map and red circles on the interferogram and phase maps. At the onset of the vortex nucleation, four of them are nucleated in the wake of the defect, but two of them (circled with dotted lines) merge together in less than 2 ps. It indicates that the size of the obstacle is probably large enough to nucleate a vortex pair, the core diameters of which are measured to be in the range $2 \mu \mathrm{m}$ to $3.3 \mu \mathrm{m}$ (depending on the vortex under scrutiny and the line profile axis), but too small to allow the nucleation of four vortices. The merging of the two central vortices may also be due to the local disorder landscape. The remaining vortex pair flows downstream, and we track its motion (white dots on the density map) for $10 \mu \mathrm{m}$, until the decay of the polariton population. Whereas in atomic condensates the vortex trajectories are closed loops because of the trapping potential ${ }^{17}$, the polariton vortices are free to propagate in the microcavity plane. The snake-like nature of their tracks is due to the local disorder landscape, and the slight right turn 


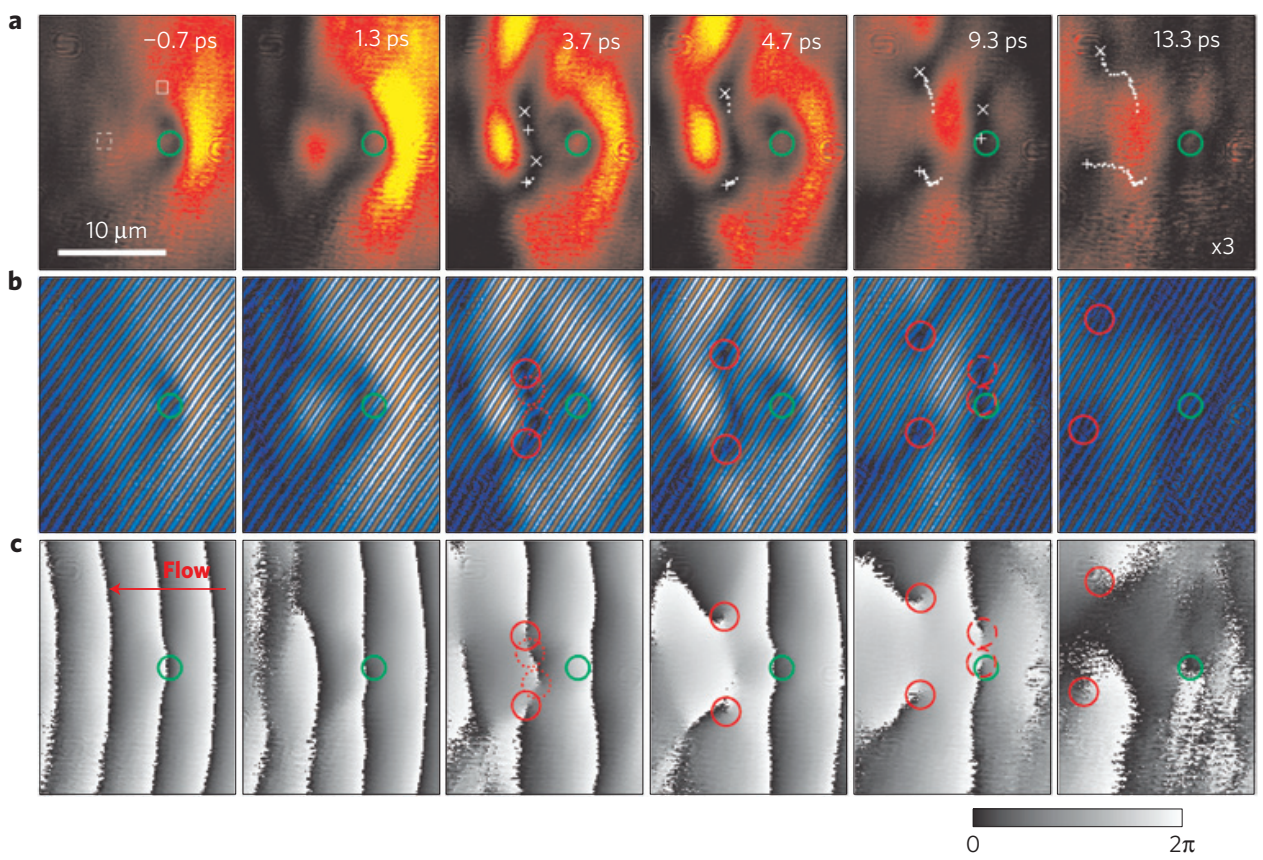

Figure $\mathbf{3}$ | Vortex dynamics. Experimental images of the scattering of the polariton wave packet on the structural defect (the position of which is indicated by a green circle). The wave packet propagates towards the left with an initial momentum of $1.2 \mu \mathrm{m}^{-1}$. The three rows show $\mathbf{a}$, the polariton density, $\mathbf{b}$, the fringes of the measured interferogram, in a saturated colour scale to facilitate the observation of the vortices, and $\mathbf{c}$, the polariton phase. First column ( $-0.7 \mathrm{ps}$ ): the phase structure is fully imposed by the excitation pulse, preventing the formation of vortices. Second column (1.3 ps): the polariton wave packet starts to feel the effect of the obstacle, resulting in a zone of minimal polariton density in the wake of the obstacle and a bending of the polariton wavefront. Third column (3.7 ps): nucleation of vortices in the wake of the obstacle. Vortices are indicated by white markers $(x$ for vortex, + for anti-vortex) on the density plot and are circled in red on the fringes and phase plots. Dotted circles indicate short-lived vortices. Fourth, fifth and sixth columns (from 4.7 to $13.3 \mathrm{ps}$ ): motion of the long-lived vortex pair. Previous vortex positions are indicated by white dots on the density plots, allowing one to follow the vortex motion. Dashed circles in the fifth column ( $9.3 \mathrm{ps})$ indicate the position of a new vortex pair which moves only a few micrometres before disappearing because of the decay of the polariton population. For the sake of visibility, density values are multiplied by a factor three in the last density plot. The average excitation power is $4 \mu \mathrm{W} \mu \mathrm{m}^{-2}$. This figure corresponds to Supplementary Movies SM1 (fluid density) and SM2 (fluid phase).

is attributed to the microcavity wedge, which provides a global potential gradient towards this direction. It is also interesting to note the additional vortex pair created at a delay of 9 ps (visible in the fifth column, dashed circles). This pair propagates only a few micrometres and then disappears in the noise due to signal decay. It does not allow one to define a shedding frequency, as this latter is expected to depend on the fluid density ${ }^{2,3}$, which varies with time in our experiment.

\section{Determining experimental conditions for vortex nucleation}

To determine the nucleation conditions in terms of polariton density and fluid velocity, we performed the same experiment with different excitation angles and powers. In theoretical predictions $s^{2,4,36}$ different flow regimes are observed, depending on the Mach number. Turbulence is expected in the wake of the obstacle when the local velocity on the perimeter of the impenetrable obstacle becomes supersonic. The original work of Frisch et al. ${ }^{2}$ also predicts that this critical velocity is attained on the obstacle perimeter when $v / c_{\mathrm{s}} \sim 0.4$ far from the defect, in a homogeneous and steady flow. In our case, it is not possible to use such a criterion, as we have a finite-size, time-dependent population, and most likely a penetrable obstacle. The only way to determine the experimental conditions for vortex nucleation is therefore to look at the local fluid velocity and sound velocity on the obstacle perimeter. Whereas the fluid velocity vector field can be directly extracted from the polariton field phase gradient, the local speed of sound can be obtained from the density map (see Methods for details). It is therefore possible to estimate the value of the local Mach number $v / c_{\mathrm{s}}$ on the obstacle perimeter along the dynamics. These values, computed in the area delimited by the plain white square in Fig. 3a, are displayed in Fig. 4b (red curve). It shows that a value of $v / c_{\mathrm{s}} \sim 1$ is obtained on the obstacle perimeter at the onset of the vortex nucleation mechanism.

Reducing the excitation angle, we have probed different wavepacket momenta, and observed the nucleation of vortices in the wake of the obstacle down to a critical initial fluid momentum of $0.6 \mu \mathrm{m}^{-1}$. Below this characteristic momentum, the wave packet passes the obstacle without any visible perturbation (see Supplementary Fig. S1). The values of the Mach number on the obstacle perimeter for this regime are plotted in blue in Fig. 4b, for comparison with the vortex nucleation regime. They show that the flow remains mostly subsonic during the major part of its dynamics. Finally, we have also varied the average excitation power and observed a threshold (at $0.04 \mu \mathrm{W} \mu \mathrm{m}^{-2}$ ) under which no vortices are nucleated in the wake of the obstacle. Instead, parabolic backscattering standing waves are visible on the polariton density maps (see Supplementary Fig. S2), and a Rayleigh ring is visible in the Fourier plane, as shown in Fig. 2b. This corresponds to a standard elastic scattering process, where the flow is supersonic, as the polariton density - and consequently the sound velocity-is very low. In this low-density limit (also called the linear regime), the interaction energy is negligible with respect to the kinetic energy.

\section{Insights from numerical simulations}

These three behaviours—-vortex pair nucleation for a $1.2 \mu \mathrm{m}^{-1}$ momentum wave packet, almost unperturbed flow in a $0.6 \mu \mathrm{m}^{-1}$ momentum wave packet and standard Rayleigh scattering in the 

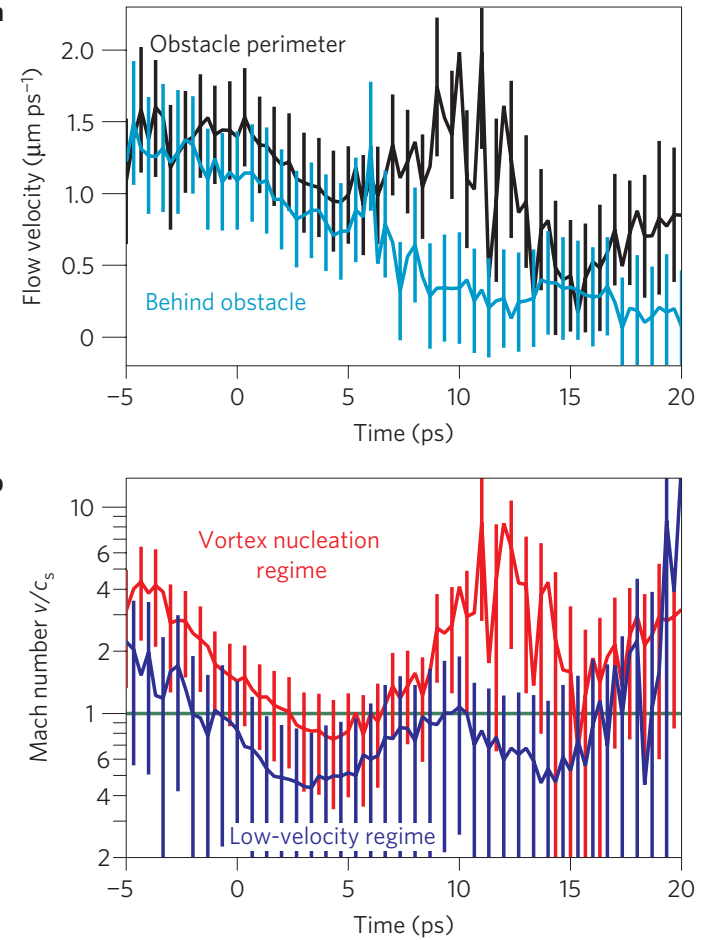

Figure 4 | Nucleation conditions in experiments. a, Experimentally measured fluid velocity $v$ for the vortex nucleation regime. Cyan curve: velocity measured behind the obstacle (in the area delimited by the dashed white square in Fig. 3a). Black curve: velocity measured on the obstacle perimeter (in the area delimited by the plain white square in Fig. 3a). These values are extracted from the polariton field phase gradient (see Methods for details). b, Mach number $v / c_{s}$ (red curve) for the vortex nucleation regime (as in Fig. 3); (blue curve) for the low-velocity regime (as in Supplementary Fig. S1). The local value of the sound velocity $c_{s}$ is extracted from the polariton field density (see Methods for details). The green line on $v / c_{s}=1$ indicates the limit between subsonic flow (below the line) and supersonic flow (above the line).

low-density flow-are extremely well reproduced by the numerical simulations (see Supplementary Figs S3, S4, S5), which take into account the pulsed excitation, the finite spot size and the exponential decay of the polariton population (see Methods). A snapshot of the computed phase profile is displayed in Fig. 5a, for the vortex nucleation regime, at the onset of the first vortex pair nucleation. Local values of the Mach number are represented by coloured lines, with a thick green line for $v / c_{s}=1$. To compare the experimental findings of Fig. $4 \mathrm{~b}$, we plot in Fig. $5 \mathrm{~b}$ the time evolution of the Mach number in a small region close to the equator of the obstacle (small black and white circle in Fig. 5a), using simulation parameters corresponding to the three flow regimes described previously (vortex nucleation regime, lowvelocity regime and low-density regime). Whereas the low-density experiment always lies in the supersonic region (black curve), the high-density experiments (blue and red curves) remain subsonic for a significant part of their dynamics. Similarly to the experimental findings, the low-velocity regime (blue curve) remains subsonic for a longer time than the vortex nucleation regime (red curve). Moreover, consistently with the argument originally developed by Frisch et al. ${ }^{2}$ for the transition to turbulence in a superfluid, we find in the simulation that the phase accumulation resulting in vortex nucleation starts at the precise time when the fluid velocity becomes equal to the sound speed (when $v / c_{\mathrm{s}}=1$ ) on the obstacle equator. The nucleation of the vortex pair is just after this event; the higher the initial velocity, the closer the vortex nucleation

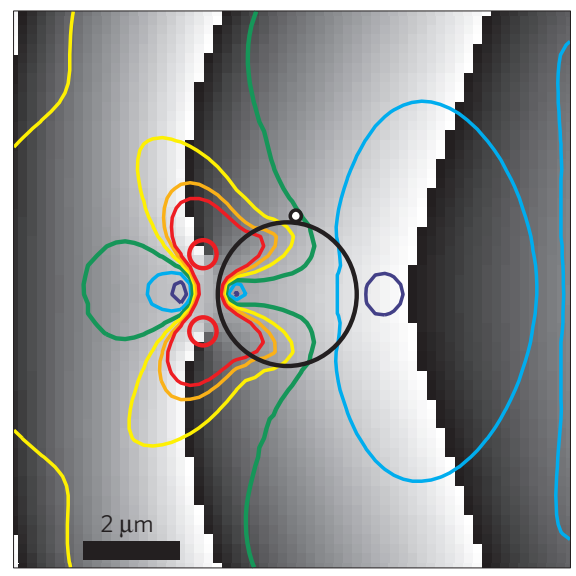

b

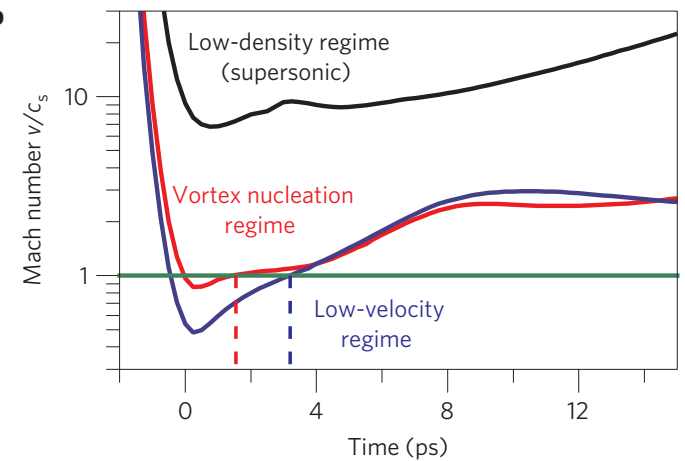

Figure 5 | Nucleation criterion: numerical evidence. a, Numerical simulation (see Methods for details) of the phase profile, with simulation parameters corresponding to the vortex nucleation regime at the onset of vortex nucleation. The obstacle is indicated by a black circle and the flow is directed to the left. The thick red circles show the vortex positions.

Coloured lines indicate lines of equal Mach number. The thick green line indicates a local Mach number of 1 , blue lines indicate a local subsonic flow $\left(v / c_{s}<1\right)$, yellow to red lines indicate a local supersonic flow $\left(v / c_{s}>1\right)$. b. Evolution of the Mach number at the equator of the obstacle (small black and white circle of Fig. 5a), using simulation parameters corresponding to the vortex nucleation regime (red curve), to the low-velocity-mainly subsonic-regime (blue curve) and to the low-density-linear-regime (black curve). The phase accumulation starts when the fluid velocity crosses the sound velocity $\left(v / c_{s}=1\right)$ on the obstacle equator (dashed lines) resulting in the systematic nucleation of vortices.

to the initial phase accumulation. The vortices are dragged away from the obstacle at later times, in a time range corresponding to the experimental findings. Finally, the numerical simulations allow one to see the role of the polaritonic nonlinearities in the nucleation process. Indeed, there are no vortices nucleated in the wake of the obstacle if the interaction constant is set to 0 . The hydrodynamic nucleation process can therefore be differentiated from linear optical processes, such as the generation of vortex lattices, whenever three or more plane waves interfere.

\section{Conclusion}

Our experiment demonstrates the great potential of semiconductor microcavities for the study of turbulence in quantum gases. The key advantages are the direct optical access to the polariton field (in both real and momentum spaces), the absence of a trapping potential and operation at cryogenic temperature (and possibly even at room temperature in state-of-the-art nitride-based microcavities ${ }^{37}$ ). The ability to control polariton properties opens the way to subsequent breakthrough experiments, such as the scattering of a wave packet on engineered obstacles of different sizes and shapes ${ }^{38,39}$, which 
would provide the possibility to address the quantum counterpart of Bénard-Von Kármán vortex streets and fully turbulent regimes ${ }^{7}$.

\section{Methods}

Sample. The sample is a GaAs $\lambda$ microcavity sandwiched between two distributed Bragg reflectors (DBR) with 22 (21) AlAs/GaAs pairs for the bottom (top) DBR, and one $8 \mathrm{~nm}$ thick InGaAs QW placed at the anti-node of the cavity field. It is held in a cold finger cryostat at liquid helium temperature. We measured a Rabi splitting of $3.5 \mathrm{meV}$ and a cavity mode quality factor of $Q=7 \times 10^{3}$. The obstacle consists of a structural defect in the microcavity plane. It is most likely penetrable. Its characteristic size can be estimated from Supplementary Fig. S1 to be $2 \mu \mathrm{m}$ in the direction of the flow and $5 \mu \mathrm{m}$ in the direction transverse to the flow.

Experimental set-up. The polariton population is created using a pulsed Ti:sapphire laser. The circularly polarized laser pulse is spectrally filtered to form a $1 \mathrm{meV}$ broad, $3 \mathrm{ps}$ long pulse, which is then split into one excitation pulse and one reference pulse. The reference pulse is directed through a telescope for spatial filtering and wavefront tuning, and incident at a slight angle on the CCD, to serve as a phase reference ${ }^{40}$. The excitation pulse is passed through a delay line and focused on the back of the sample using a $25 \mathrm{~cm}$ focal length camera objective, providing a Fourier limited $25 \mu \mathrm{m}$ diameter excitation spot. An oblique excitation angle is used to create a propagating polariton wave packet. The coherent emission is collected by means of a $0.5 \mathrm{NA}$ microscope objective in a transmission configuration. Real space or Fourier space images of the coherent emission are allowed to interfere, in a Mach-Zehnder configuration, with the reference pulse on the CCD. From the interferogram we numerically extract the amplitude and phase of the coherent emission at a time given by the delay between the excitation and reference pulses. Varying this delay allows us to probe the dynamics of the coherent polariton population in both real and momentum spaces By acquiring data over $0.2 \mathrm{~s}$, every measurement is an integration over $1.6 \times 10^{7}$ experimental realizations.

Numerical extraction of polariton amplitude and phase. To extract the polariton amplitude and phase from the recorded interferogram, we use a technique known as digital off-axis holography ${ }^{41}$. It consists of performing a two-dimensional fast Fourier transform (FFT) of the interferogram. The fringes of the interferogram provide off-axis contributions in the Fourier plane, which can be differentiated from the $c w$ contributions. Removing the $c w$ contributions and performing an inverse FFT allows one to isolate the fringes, from which the amplitude and phase are extracted, providing the full information (amplitude and phase) on the coherent polariton field.

Extraction of local fluid velocity and speed of sound from experimental data. The fluid velocity vector field is extracted from the phase gradient of the polariton field. The values of Fig. 4a are obtained by fitting the slope of the phase profile in the region of interest. The error bars take into account the standard deviation of the linear fit, as well as a systematic error coming from the determination of the phase gradient induced by the set-up alignment (see ref. 40 for how this reference phase gradient can be determined). The value of the local sound velocity is determined from the density map, originally in arbitrary units, which needs to be scaled to the blue-shift $n g$. We have access only to the spatially and temporally averaged blue-shift in the polariton dispersion, which is measured to be $0.8 \mathrm{meV}$. Assuming that the major contribution to this blue-shift comes from the beginning of the dynamics, when the maximal population density is reached, we scale the density maps to the blue-shift. This allows us to extract a rough estimation for the local speed of sound along the dynamics (it more probably gives a lower bound to its value, as the averaging of the blue-shift yields an underestimation of its value). We consider an error in the local sound velocity which takes into account the standard deviation on the averaging in the region of interest, as well as a systematic error on the scaling method, estimated to $25 \%$.

Gross-Pitaevskii equation. We theoretically investigate the quantum turbulence regime of exciton-polaritons in the mean field approximation. We solve iteratively the generalized Gross-Pitaevskii equation for the lower polariton mode $\psi$, previously introduced by Ciuti and Carusotto for exciton polaritons ${ }^{34}$

$$
\begin{aligned}
i \hbar \frac{\mathrm{d}}{\mathrm{d} t} \psi(\mathbf{r}, t)= & \left(-i \frac{\gamma}{2}+\sum_{k} \hbar \omega_{k}|k\rangle\left\langle\left. k|+g| \psi(\mathbf{r}, t)\right|^{2}\right) \psi(\mathbf{r}, t)\right. \\
& +V \psi(\mathbf{r}, t)+F_{k}(\mathbf{r}, t)
\end{aligned}
$$

The potential $V$ is constructed as a $3 \mu \mathrm{m}$ sized and $1 \mathrm{meV}$ high obstacle. Our model accounts for the dissipation of polaritons, at rate $\gamma$, and a 1 ps-long initial excitation of the system $F_{k}(r, t)$. The polariton-polariton interaction is assumed to depend linearly on the polariton density $|\psi(\mathbf{r}, t)|^{2}$ with a coefficient $g$. The lower polariton dispersion is approximated to a quadratic dispersion with effective mass $m_{\mathrm{LP}}$. The parameters used in the simulations are: $\gamma=\hbar / 15 \mathrm{ps}, g=0.01 \mathrm{meV} \mu \mathrm{m}^{2}$, $\hbar \omega_{k}=\hbar^{2} k_{\|}^{2} / 2 m_{\mathrm{LP}}$ with $m_{\mathrm{LP}}=0.7 \mathrm{meV} \mathrm{ps}^{2} \mu \mathrm{m}^{-2}$. The excitation intensity for the high excitation experiment corresponds to a maximal polariton density of $120 \mu \mathrm{m}^{-2}$ on a $20 \mu \mathrm{m}$ large spot.

Received 3 November 2010; accepted 28 February 2011; published online 3 April 2011

\section{References}

1. Bergé, P., Pomeau, Y. \& Vidal, C. Order Within Chaos: Towards a Deterministic Approach to Turbulence (Wiley, 1986).

2. Frisch, T. et al. Transition to dissipation in a model superflow. Phys. Rev. Lett. 69, 1644-1647 (1992)

3. Winiecki, T., McCann, J. F. \& Adams, C. S. Pressure drag in linear and nonlinear quantum fluids. Phys. Rev. Lett. 82, 5186-5189 (1999).

4. Winiecki, T. et al. Vortex shedding and drag in dilute Bose-Einstein condensates. J. Phys. B 33, 4069-4078 (2000).

5. Barenghi, C. F., Donnelly, R. J. \& Vinen, W. F. (eds) Quantized Vortex Dynamics and Superfluid Turbulence (Springer, 2001).

6. Aftalion, A. et al. Dissipative flow and vortex shedding in the Painlevé boundary layer of a Bose-Einstein condensate. Phys. Rev. Lett. 91, 090407 (2003).

7. Sasaki, K. et al. Bénard-von Kármán vortex street in a Bose-Einstein condensate. Phys. Rev. Lett. 104, 150404 (2010).

8. Mironov, V. A. et al. Structure of vortex shedding past potential barriers moving in a Bose-Einstein Condensate. J. Exp. Theor. Phys. 110, 877-889 (2010).

9. Raman, C. et al. Evidence for a critical velocity in a Bose-Einstein condensed gas. Phys. Rev. Lett. 83, 2502-2505 (1999).

10. Onofrio, R. et al. Observation of superfluid flow in a Bose-Einstein condensed gas. Phys. Rev. Lett. 85, 2228-2231 (2000).

11. Madison, K. W., Chevy, F., Wohlleben, W. \& Dalibard, J. Vortex formation in a stirred Bose-Einstein condensate. Phys. Rev. Lett. 84, 806-809 (2000).

12. Madison, K. W., Chevy, F., Bretin, V. \& Dalibard, J. Stationary states of a rotating Bose-Einstein condensate: Routes to vortex nucleation. Phys. Rev. Lett. 86, 4443-4446 (2001)

13. Raman, C., Abo-Shaeer, J. R., Vogels, J. M., Xu, K. \& Ketterle, W. Vortex nucleation in a stirred Bose-Einstein condensate. Phys. Rev. Lett. 87, 210402 (2001).

14. Yarmchuk, E. J., Gordon, M. J. V. \& Packard, R. E. Observation of stationary vortex arrays in rotating superfluid helium. Phys. Rev. Lett. 43, 214-217 (1979).

15. Henn, E. A. L., Seman, J. A., Roati, G., Magalhes, K. M. F. \& Bagnato, V. S. Emergence of turbulence in an oscillating Bose-Einstein condensate. Phys. Rev. Lett. 103, 045301 (2009).

16. Inouye, S. et al. Observation of vortex phase singularities in Bose-Einstein condensates. Phys. Rev. Lett. 87, 080402 (2001).

17. Neely, T. W. et al. Observation of vortex dipoles in an oblate Bose-Einstein condensate. Phys. Rev. Lett. 104, 160401 (2010).

18. Amo, A. et al. Superfluidity of polaritons in semiconductor microcavities. Nature Phys. 5, 805-810 (2009).

19. Weisbuch, C. et al. Observation of the coupled exciton-photon mode splitting in a semiconductor quantum microcavity. Phys. Rev. Lett. 69, 3314-3317 (1992)

20. Cerna, R. et al. Coherent optical control of the wave function of zero-dimensional exciton polaritons. Phys. Rev. B 80, 121309(R) (2009).

21. Nardin, G. et al. Selective photoexcitation of confined exciton-polariton vortices. Phys. Rev. B 82, 073303 (2010).

22. Saba, M. et al. High-temperature ultrafast polariton parametric amplification in semiconductor microcavities. Nature 414, 731-735 (2001).

23. Kasprzak, J. et al. Bose-Einstein condensation of exciton polaritons. Nature 443, 409-414 (2006).

24. Carusotto, I. et al. Fermionized photons in an array of driven dissipative nonlinear cavities. Phys. Rev. Lett. 103, 033601 (2010).

25. Love, A. P. D. et al. Intrinsic decoherence mechanisms in the microcavity polariton condensate. Phys. Rev. Lett. 101, 067404 (2008).

26. Lagoudakis, K. G. Coherent oscillations in an exciton-polariton Josephson junction. Phys. Rev. Lett. 105, 120403 (2010).

27. Wertz, E. et al. Spontaneous formation and optical manipulation of extended polariton condensates. Nature Phys. 6, 860-864 (2010).

28. Lagoudakis, K. G. et al. Quantized vortices in an exciton-polariton condensate. Nature Phys. 4, 706-710 (2008).

29. Lagoudakis, K. G. et al. Observation of half-quantum vortices in an exciton-polariton condensate. Science 326, 974-976 (2009).

30. Roumpos, G. et al. Single vortex-antivortex pair in an exciton-polariton condensate. Nature Phys. 7, 129-133 (2011)

31. Krizhanovskii, D. N. et al. Effect of interactions on vortices in a nonequilibrium polariton condensate. Phys. Rev. Lett. 104, 126402 (2010).

32. Sanvitto, D. et al. Persistent currents and quantized vortices in a polariton superfluid. Nature Phys. 6, 527-533 (2010).

33. Amo, A. et al. Collective fluid dynamics of a polariton condensate in a semiconductor microcavity. Nature 457, 291-295 (2009). 
34. Carusotto, I. et al. Probing microcavity polariton superfluidity through resonant Rayleigh scattering. Phys. Rev. Lett. 93, 166401 (2010).

35. Bolda, E. L. et al. Dissipative optical flow in a nonlinear Fabry-Perot cavity. Phys. Rev. Lett. 86, 416-419 (2001).

36. Pigeon, S. et al. Hydrodynamic nucleation of vortices and solitons in a resonantly excited polariton superfluid. Preprint at http://arxiv.org/abs/1006.4755 (2010).

37. Christmann, G. et al. Room temperature polariton lasing in a GaN/AlGaN multiple quantum well microcavity. Appl. Phys. Lett. 93, 051102 (2008).

38. Kaitouni, R. I. et al. Engineering the spatial confinement of exciton polaritons in semiconductors. Phys. Rev. B 74, 155311 (2006).

39. Amo, A. et al. Light engineering of the polariton landscape in semiconductor microcavities. Phys. Rev. B 82, 081301(R) (2010).

40. Nardin, G. et al. Phase-resolved imaging of confined exciton-polariton wave functions in elliptical traps. Phys. Rev. B 82, 045304 (2010).

41. Kreis, T. Holographic Interferometry - Principles and Methods (Akademie, 1996).

\section{Acknowledgements}

We would like to thank M. Wouters and T. C. H Liew for enlightening discussions. We acknowledge support by the Swiss National Science Foundation through the 'NCCR Quantum Photonics'.

\section{Author contributions}

G.N. and G.G performed the experiments. Y.L. performed the numerical simulations. F.M-G. grew the sample. G.N, Y.L and B.P. wrote the paper. B.D-P. supervised the project. All authors contributed to numerous discussions and data analysis.

\section{Additional information}

The authors declare no competing financial interests. Supplementary information accompanies this paper on www.nature.com/naturephysics. Reprints and permissions information is available online at http://npg.nature.com/reprintsandpermissions. Correspondence and requests for materials should be addressed to G.N. 